\title{
The Contributions of Language and Behavioral Synchrony in Developing Affect Regulation in High-Risk Children
}

\author{
Angela Adger-Antonikowski' ${ }^{1}$, Leslie F. Halpern ${ }^{2}$ \\ ${ }^{1}$ Department of Pediatrics, Albany Medical College, Albany, USA \\ ${ }^{2}$ Department of Psychology, University at Albany-SUNY, Albany, USA \\ Email: Antonia@mail.amc.edu
}

Received 20 February 2014; revised 20 March2014; accepted 28 March 2014

Copyright (C) 2014 by authors and Scientific Research Publishing Inc.

This work is licensed under the Creative Commons Attribution International License (CC BY). http://creativecommons.org/licenses/by/4.0/

(c) (†) Open Access

\section{Abstract}

Biological vulnerability translates into behavioral risks that often extend beyond the infancy period. Pre-term children born low birth weight are at risk for behavioral and emotional difficulties, however, little is known about how these difficulties manifest and interact with biological risk and environmental factors. This study examined the extent to which children's language and dyadic relationship factors facilitate affect regulation and behavior problems in low birth weight children (LBW). Sixty-eight preschool-age children and their mothers participated in the study. Children were administered an assessment of cognitive functioning in which verbal ability was examined, and engaged in a laboratory frustration task designed to elicit negative affect to assess emotion regulation. Results revealed correlations among verbal ability, behavior problems, behavioral synchrony, and emotion regulation. Group differences were found in verbal ability and positive affect in which LBW children demonstrated lower verbal ability scores and less positive affect than full-term children. For the full sample, behavior synchrony moderated the relationship between verbal ability and negative emotion regulation. The results provide evidence of the importance of the functional role of language and dyadic relations in the development of affect regulation and behavior problems. The results also elucidate the importance of integrating social emotional information in developing interventions for pre-term children.

\section{Keywords}

Prematurity, Medical Vulnerability, High-Risk, Parenting, Emotion, Behavior Problems

\section{Introduction}

The development of emotion regulation skills is central to the psychological adjustment of children and adoles-

How to cite this paper: Adger-Antonikowski, A. and Halpern, L.F. (2014) The Contributions of Language and Behavioral Synchrony in Developing Affect Regulation in High-Risk Children. Open Journal of Medical Psychology, 3, 271-280. 
cents [1] [2]. There are multiple factors that contribute to the attainment of emotion regulation skills. These include biologically-rooted behavioral predispositions, such as temperament [3], and biologically-based deficits that are the byproduct of neonatal insults, injuries, or high-risk birth status [4]. The biological factors exert an influence on developmental outcomes such that they impact how children experience their environment and the responses from persons in that environment including caregivers. Pre-term children born low birth weight (LBW) are a group of children identified to demonstrate deficits in emotion regulation as well as other domains of functioning due in part to biologically rooted mechanisms. In terms of emotion and behavior, LBW children demonstrate fewer positive affect interactions, more negative arousal, and fewer positive affect cues in laboratory paradigms [5] [6]. LBW children not only have difficulties initiating and maintaining emotion regulation, but their cues for arousal are often misconstrued by caregivers. The contribution of affective states and temperamental dispositions make affective synchrony and subsequent emotion regulation mastery difficult for pre-term/caregiver dyads.

\section{Emotion Regulation Deficits and Language Ability}

The preschool period signals a transition to more self-directed regulation strategies. During these years, the developing child has not mastered the effective use of complex emotion regulation skills; however, due to an increase in language ability, the preschool child engages in more compliance and self-control behaviors which aid in the development of emotion regulation [7]. Language facilitates this process by allowing children to internalize experiences and to better modulate negative emotions by offering a larger repertoire of regulation strategies. These strategies include self-talk and the solicitation of emotion-coping strategies from caregivers. There are numerous examples of research that suggest there is a relationship between deficits in language ability and behavior problems [8]-[12]. For young children with language skills deficits, emotion regulation is likely more difficult. Deficits in language ability may not preclude adequate emotion regulation for young children, however, if caregivers are responsive to their children's emotional signals, and provide direct instruction regarding emotion regulation or model adequate emotion regulation for their children. Emotion regulation therefore is, in part, a manifestation of a caregiver's ability to respond appropriately to displayed affect and to motivate positive interactions within the dyad [5]. In turn, the extent to which children clearly express emotion cues will greatly affect caregivers' ability to respond to their children. Further, children's emotion regulation skills are likely impacted by both language capabilities and exposure to models who demonstrate emotion management behaviors or who provide direct instruction regarding emotion regulation. For pre-term children, the ability to model affect regulation, and caregivers' responsiveness to affective cues are particularly salient to emotional development.

\section{Interactional Synchrony and LBW Children}

Few studies have examined the contribution of behavioral synchrony, or the temporal sequencing of behavioral or affective states amongst dyads and language ability, to child behavior problems and emotions. The impact of birth status on these relationships is rarely examined; however, there is some evidence that pre-term children with speech delays have more maladaptive interaction patterns as opposed to pre-term children with better language skills [13].

Research has demonstrated that the quality of social, dyadic interactions impacts the cognitive and language development of the at-risk infant [14]. In addition, differences in synchrony are thought to underlie later differences in language development and behavior problems in pre-term and full-term children because more synchronous relationships provide an organization for emotional and cognitive development [15]. Due to difficulties in bringing the pre-term infant to optimal levels of arousal, and difficulties in accurately interpreting preterm infants' affective cues, caregivers of pre-term infants may display less positive affect and feel less efficacious and more distressed in their caregiving role [16]. The salience of understanding the biological risks associated with pre-term birth and difficulties in dyadic relationships later in development underscores the importance of synchrony in early childhood, particularly with pre-term children. Research on the interactions of children born prematurely and their caregivers has focused largely on infancy, with little known about dyadic interactions later in childhood despite evidence that behavior and emotional difficulties persist into childhood and beyond. The few studies that have examined dyadic interactions beyond the infancy period have found that there are stable patterns of interaction that impact children's developing cognitive and social skills [17] [18].

Deficits in adequate modeling of emotion regulation may occur more often in LBW and VLBW dyads due to 
the bidirectional influences of child and parenting factors. Further, children with deficits in emotion regulation due to biological vulnerabilities (i.e., LBW children), may differ in the manner in which they utilize language. Low birth weight and very-low birth weight children provide a unique opportunity to examine the impact of language and caregiver modeling of emotion regulation on children's emotional development and behavior problems due to the unique relationships present amongst biological vulnerabilities and caregiver influences. Further, because the biological vulnerability of the pre-term child extends into early childhood and beyond, the understanding of how emotion regulation manifests is paramount to developing interventions to improve preterm children's outcomes.

The overall purpose of this research was to examine the contributions of children's language ability and dyadic behavioral synchrony to young children's emotion regulation and behavior problems. Toward this end, this study examined the degree to which language and dyadic behavioral synchrony, in dyads where emotion regulation has been demonstrated to be problematic, functions to facilitate children's emotion regulation during a frustrating event. It was hypothesized that dyadic behavioral synchrony will moderate the relationship between verbal ability and emotion regulation such that low verbal skills will not preclude adaptive regulation. Further, it was hypothesized that LBW children will experience more deficits in emotion regulation and verbal skills as compared to children born full term, however, LBW-mother dyads that demonstrate high levels of synchrony, irrespective of the child's verbal skills, will demonstrate better emotion regulation.

\section{Methods}

\subsection{Participants}

The participants in this study were 68 preschool-age children ( $\mathrm{M}$ age $=4$ years, 3 months, $\mathrm{SD}=5$ months) and their mothers who were recruited for a larger project studying the developmental outcomes of preschoolers born prematurely. Participants recruited as LBW were born at less than 2500 grams (5.51 pounds) and born at equal or less than 36 weeks gestational age. Full-term preschoolers were at least 2501 grams (5.52 pounds) at birth and at least 37 weeks gestational age. All participating families were English speaking and mothers had to be at least sixteen years of age and not have used illicit substances during their pregnancy.

Forty LBW children between the ages of 3 and 4 years of age ( $M=4$ years, 5 months, $\mathrm{SD}=5$ months $)$ and their mothers participated in this study. The mean birth weight was 1188.66 grams or 2.62 pounds ( $\mathrm{SD}=379.53$ grams) with weight ranging from 545 grams (1.20 pounds) to 2496 grams (5.50 pounds). Mean gestational age at birth was 29.40 weeks $(\mathrm{SD}=2.94)$ with gestational age ranging between 24 and 36 weeks, and their mean Hobel Risk Score was $30.00(\mathrm{SD}=14.31)$. Mothers reported their children as African-American, $8 \%(\mathrm{n}=3)$, European-American (Caucasian), 90\% $(\mathrm{n}=36)$, and Bi-racial, 2\% $(\mathrm{n}=1)$. Fifty-seven percent of the LBW sample were female $(\mathrm{n}=23)$. The mean age of the mothers of the LBW children was 35.82 years $(\mathrm{SD}=7.28)$, and $76 \%$ reported living in married households, $10 \%$ reported living in single households, $3 \%$ reported being separated, and $11 \%$ reported they were living together with a partner. Mean family income reported by LBW mothers was $\$ 59,124$ ( $\mathrm{SD}=\$ 30,049)$ with annual income ranging from $\$ 4392$ to $\$ 110,000$. Nineteen percent of mothers reported partially completing or completing high school, $65 \%$ reported either completing college or attending some college, and $16 \%$ reported either completing graduate level education or having specialized training.

The full term sample was comprised of twenty-eight children between the ages of 3 and 4 years of age $(\mathrm{M}$ age $=$ 4 years, 1 month, $\mathrm{SD}=5$ months) and their mothers. The mean birth weight of full-term children was 3335.30 grams or 7.35 pounds $(\mathrm{SD}=482.09$ grams) with weight ranging from 2735.16 grams ( 6.03 pounds) to 4545 grams (10.02 pounds). Mean gestational age of the full-term sample was 39.43 weeks $(\mathrm{SD}=1.23)$ with gestational age ranging from 37 and 42 weeks. Mothers reported their children as Asian, 4\% $(n=1)$, African-American, 10\% ( $\mathrm{n}=3)$, Bi-racial, 7\% $(\mathrm{n}=2)$, and European-American (Caucasian), 79\% $(\mathrm{n}=22)$. Fifty-seven percent of full-term children were female $(n=16)$. The mean age of the mothers of full-term children was 35.85 years $(\mathrm{SD}=5.40)$, and $75 \%$ reported living in married households, $11 \%$ reported living in single households, $10 \%$ of mothers reported being separated, and $4 \%$ reported residing in divorced households. The mean family income for full-term mothers was $\$ 81,788$ ( $\mathrm{SD}=\$ 34,338$ ) with annual income ranging from $\$ 28,000$ to $\$ 160,000$. Seven percent of mothers reported partially completing or completing high school, $56 \%$ reported partially completing or completing college, and $37 \%$ reported either completing graduate level education or having specialized training. 


\subsection{Procedures}

LBW participants were recruited through a Developmental/Behavioral pediatrician's practice providing follow-up services to pre-term children born at one of two hospitals in Upstate NY. Full-term participants were recruited through area daycare centers, preschools, and the pediatric outpatient program of one of the two hospitals where the pre-term children were born.

Laboratory Visit Information. Dyads participated in two home visits prior to participating in a series of laboratory procedures as part of a larger project examining preschool children's development. During the laboratory visit, a cognitive assessment was administered first followed by four laboratory procedures which included a frustration task. For the purposes of the current research study, only the frustration task will be discussed further.

Frustration Task. Children were seated at the head of a table and their mothers were seated in a larger chair perpendicular to them. Children were given a toy stamp to play with for one minute. At the end of one minute, the research assistant retrieved the toy stamp from the child and placed it in a child-proof container with other small attractive toys. The child was then asked to retrieve the toy stamp if they wanted to. The toy stamp remained in the container for four minutes at which time the research assistant retrieved the toy stamp, and the child was able to continue stamping for one additional minute. At the end of the one-minute, the child was able to choose a toy from among other toys that were in the child-proof container. The child was engaged in the frustration paradigm for a total of six minutes. Prior to beginning the frustration task, mothers were told their child would be engaged in a frustrating situation. Mothers were encouraged not to explicitly open the container and were directed to magazines on the table should they choose to read. The frustration task was video-taped and coded at a later time.

\subsection{Measures}

General Information. Mothers were asked to complete a general information form. The general information form asked mothers to report on demographic information (e.g., child ethnic background, marital status, mother age, education, and family income) as well as child medical information such as gestational age, birth weight, birth order, neonatal hospitalizations and maternal health history. Records for LBW children were obtained by the pediatrician.

Language Ability. Children's cognitive ability was assessed using the Stanford Binet-Fourth Edition. [19] Standard Area Scores (SAS) were generated for each of the subtests to comprise scaled SAS scores. As an index of verbal ability, the Verbal Reasoning content area SAS was used in data analyses for the purpose of this study. The Verbal Reasoning content area score reflects children's performance on three subtests: Vocabulary, Comprehension, and Absurdities. The Verbal Reasoning subtests are reliable with internal consistency reliabilities of .87 reported for each subtest. The Vocabulary, Comprehension, and Absurdities subtests are highly correlated with the Composite score $(r=0.81 ; r=0.76 ; r=0.72$, respectfully).

Child Behavior Problems. Child behavior problems were assessed using the Child Behavior Checklist, or CBCL $-1.5-5$ [20]. Parents are asked to rate their child's behavior on a three-point Likert scale over the course of the previous two months. This measure yields an assessment of child behavior on multiple syndrome subscales, two broad-band scales (Internalizing and Externalizing problems), and an overall Behavior Problem Score. The Internalizing and Externalizing broad-band scales, and the syndrome subscales of Emotionally Reactive and Attention Problems were used as indicators of behavior problems in the current study. The T-scores were used when examining Internalizing and Externalizing problems, and the raw scores were used when examining the syndrome scales of Attention Problems and Emotionally Reactive Problems.

Coding of Behavioral Synchrony. Dyadic behavioral synchrony was assessed using contingency coding and frequency ratings during the four-minute frustration task. Behavioral synchrony coding was based upon an interaction cycle that involves the child's initiation of a request, the mother's response to the request, and the child's subsequent response. The child's "bids" for attention or initiation of a request involved any verbal statement made by the child to secure the mother's attention with respect to the frustration container-task. Child behaviors that were coded included, Task-Oriented Attention Seeking, Proximity Seeking, Distraction, or Task-Oriented Attention Seeking directed toward the laboratory assistant. Mother responses to each instance of child initiation were evaluated in terms of the frequency of empathic and non-empathic responses (i.e., Empathic Verbalizations, Non-Empathic Verbalizations and Proximity-encouraging Behavior), and in terms of task-related responses (i.e., Task-oriented Verbalizations and Behaviors, Distraction, and Ignoring). The child's responses to the mother's 
response were coded in terms of Task-Oriented Attention Seeking, Proximity Seeking, Distraction, TaskOriented Attention Seeking directed toward the laboratory assistant, or Ignoring. For this study, Behavioral Synchrony is defined as the degree to which the dyad is mutually engaged in similar behavioral goals as set forth by the mother. A Behavioral Synchrony Index was derived by computing the proportion of interaction cycles in which the child ends the cycle engaged in a behavior set forth by the mother in her response to the child's initial bid for attention. It is an objective index derived from visually inspecting the behaviors recorded on the coding sheet. For example, at the end of the response phase, if the child is engaged in task-oriented behavior after the mother has facilitated behavior through her task-oriented or empathic verbalizations, the dyad is noted for synchrony. Similarly, if the mother engages in distraction and the child is rated as also engaging in distraction in the response phase, the dyad is noted for synchrony. If the mother ignores the child's behavior and the child engages in attention seeking, the dyad is not noted for synchrony as the child does not have the opportunity to engage in the behaviors that are of interest in this study. A higher proportion score of synchrony is indicative of greater behavioral synchrony.

Coding of Emotion Regulation. Emotion regulation was assessed at two points during the interaction cycle; first when the child attempted a bid for caregiver attention and at the end of the cycle, when the child responded to the mother's behavior. The child was assessed in terms of demonstrating joyful, sad, angry, and neutral affect. Joy, sadness, and anger intensity was rated on a 3-point scale, with 1 being the lowest level of intensity and 3 being the highest level. Once the child demonstrated a particular emotion, the intensity of the emotion was rated.

For the current study, emotion regulation is defined as the degree to which the child is able to maintain a positive or neutral affective state, or down-regulate negative affect. The mean intensity of expressed emotion was calculated during the Child Initiation and Child Response phases of the interaction cycles for sadness and anger, and the change in mean intensity within emotion was examined as an indicator of negative emotion regulation. The mean intensity of joy was also calculated. The mean intensity of affect score from initiation was subtracted from the mean intensity of affect score at response for sadness and anger to create a mean change score for each expressed affect. Mean changes in sadness and mean changes in anger were then collapsed to represent a mean change in negative affect or negative emotion regulation score. This negative emotion regulation score was later used in regression analyses.

The mean change score for joy intensity and the mean change score for the proportion of neutral affect were converted to z-scores and collapsed to represent a mean change in positive affect/neutral or positive emotion regulation score. The change in the mean intensity of joy from Child Initiation to Child Response was combined with the change in the proportion of neutral affect from Child Initiation to Child Response to represent a positive emotion regulation score. The mean intensity of emotion at the start of interaction cycle was subtracted from the mean intensity of emotion the end of the interaction cycle to represent a mean change in emotion. Scores for emotion regulation could range from negative, indicating poor emotion regulation or an increase in negative emotion from initiation to response, to positive, indicating better emotion regulation or a down regulation of negative emotion from initiation to response.

\subsection{Reliability}

Two undergraduate research assistants and the first author of this study completed the behavior and emotion coding procedures. Coders were trained by the first author and once the coders reached 0.80 reliability, they were able to begin coding videotapes independently. Each individual coded $50 \%$ of the videotapes with a $20 \%$ overlap between the coders. Inter-rater reliability was computed for $20 \%$ of the sample by calculating interclass correlation coefficients. Reliability estimates for this study ranged from $0.74-0.83$. Reliability for the behaviors that were coded was 0.81 for total bids of attention and 0.74 for total instances of behavioral synchrony. With respect to the emotion coding, reliability for proportion of joy was 0.77 , proportion of sadness was 0.77 , proportion of anger was 0.79 , and proportion of neutral affect was 0.74 . Reliability for mean level of joy 0.80 , mean level of sadness 0.82 , and mean level of anger was 0.83 .

\section{Results}

\subsection{Preliminary Data}

Prior to statistical analyses, all study variables were examined for out-of-range values, missing data, outliers, 
normality, and multicollinearity. All variables were within specified ranges.

Tests for group differences in demographic variables. Preliminary analyses were conducted to test for potential demographic covariates. T-tests were used to assess group differences in continuous variables such as parents' age, family income, and child age. Bonferroni correction procedures were used to correct for inflated alpha levels associated with multiple t-test comparisons. Chi-square analyses were used to assess group differences in demographic variables that were categorical including ethnicity, gender, marital status and parent education. T-tests revealed that the LBW children were older than full-term children, $t(65)=2.92, p=0.005$. The only other demographic variable found to differ between the two groups was family income, which was higher for the full-term group, $\mathrm{t}(65)=-2.78, \mathrm{p}=0.007$.

Descriptive statistics. Means and standard deviations are reported in Table 1 for verbal ability, behavior synchrony, behavior problem scores, and emotion regulation. One-way analyses of covariance (ANCOVAs) with income and child age included as covariates, were conducted to examine group differences in verbal ability, positive and negative emotion regulation, behavioral synchrony, and behavior problems. A significant group difference emerged for verbal ability such that full-term children had higher verbal ability scores compared to pre-term children, $\mathrm{F}(1,59)=8.06, \mathrm{p}<0.01$, and this difference represented a small effect (Partial Eta Squared $=$ 0.12 ). There were no significant differences between the LBW and full-tem children in positive and negative emotion regulation, behavior synchrony, or behavior problem $(\mathrm{p}>0.05)$.

Group differences in the intensity of joy, sadness, and anger affect at initiation and response which was tested using $2 \times 2$ mixed ANCOVA's, with group as the fixed factor and affect as the repeated factor. Income and child age were included as covariates in the analysis. The results were significant for the analysis testing joy intensity. A main effect for group was present when examining joy during the response phase such that full-term children demonstrated a greater intensity in joy in the response phase than did pre-term children, $\mathrm{F}(1,58)=5.32$; $\mathrm{p}<0.05$.

Correlations. Pearson product-moment correlations were used to assess the intercorrelations among verbal ability, behavioral synchrony, positive and negative emotion regulation, and behavior problem scores for the combined sample. Increases in behavioral synchrony were related to poorer emotion regulation. In terms of behavior problems, higher levels of verbal ability were related to lower attention problem, emotion reactivity and internalizing and externalizing scores.

Pearson product-moment correlations were also used to assess the intercorrelations among verbal ability, behavioral synchrony, positive and negative emotion regulation, and behavior problems for the LBW (Table 2) and full-term samples (Table 3) separately. For the full-term group, higher verbal ability scores were related to lower attention, emotional reactivity and externalizing scores (Table 4). Better positive emotion scores were also related to lower attention problems scores. This pattern was different for the LBW group, however. Specifically, for the LBW group higher positive affect scores were related to lower negative reactivity scores. For the LBW sample, Hobel risk scores were assessed in terms of correlations with primary variables. Children's Hobel

Table 1. Means and standard deviations of child behavior problems, verbal ability and behavioral synchrony.

\begin{tabular}{|c|c|c|c|c|}
\hline & \multicolumn{4}{|c|}{ Mean (SD) } \\
\hline & Pre-Term & Full-Term & Minimum & Maximum \\
\hline Externalizing & $47.44(11.46)$ & $44.25(8.16)$ & 28.00 & 73.00 \\
\hline Internalizing & $49.82(12.60)$ & $47.00(7.58)$ & 33.00 & 77.00 \\
\hline Attention Problems & $2.68(2.60)$ & $1.46(1.51)$ & 0.00 & 9.00 \\
\hline Emotion Reactivity & $3.03(3.23)$ & $1.71(1.67)$ & 0.00 & 12.00 \\
\hline Verbal Ability (SB-IV) & $105.28(17.22)$ & $114.79(7.13)$ & 45.00 & 129.00 \\
\hline Behavioral Synchrony & $0.48(0.33)$ & $0.57(0.54)$ & 0.00 & 3.00 \\
\hline Negative emotion regulation & $-0.03(0.87)$ & $-0.17(1.05)$ & -4.1 & 2.0 \\
\hline Positive emotion regulation & $0.08(1.15)$ & $-0.11(1.02)$ & -2.5 & 3.7 \\
\hline
\end{tabular}


Table 2. Intercorrelations among verbal ability, behavioral synchrony, emotion, and behavior problems for combined sample.

\begin{tabular}{|c|c|c|c|c|c|c|c|c|}
\hline & 1 & 2 & 3 & 4 & 5 & 6 & 7 & 8 \\
\hline 1. Verbal Ability & - & 0.15 & -0.04 & 0.09 & $-0.46^{* *}$ & $-0.33^{* *}$ & $-0.27^{*}$ & $-0.33^{* *}$ \\
\hline 2. Behavioral Synchrony & & - & -0.04 & $-0.35^{* *}$ & 0.13 & -0.00 & 0.02 & 0.08 \\
\hline 3. Positive Emotion Regulation & & & - & 0.17 & $-0.28^{*}$ & -0.14 & -0.08 & $-0.24^{*}$ \\
\hline 4. Negative Emotion Regulation & & & & - & -0.13 & -0.02 & -0.02 & -0.15 \\
\hline 5. Attention Problems & & & & & - & $56^{* *}$ & $48^{* *}$ & $0.75^{* *}$ \\
\hline 6. Emotional Reactivity & & & & & & - & $0.89^{* *}$ & $0.74^{* *}$ \\
\hline 7. Internalizing & & & & & & & - & $0.73^{* *}$ \\
\hline 8. Externalizing & & & & & & & & - \\
\hline
\end{tabular}

Table 3. Intercorrelations among verbal ability, behavioral synchrony, emotion, and behavior problems for LBW children (n $=40)$.

\begin{tabular}{|c|c|c|c|c|c|c|c|c|}
\hline & 1. & 2. & 3 & 4 & 5 & 6. & 7. & 8. \\
\hline 1. Verbal Ability & - & 0.05 & 0.01 & 0.21 & $-0.51^{* *}$ & $-0.35^{*}$ & -0.27 & $-0.35^{*}$ \\
\hline 2. Behavioral Synchrony & & - & 0.18 & 0.05 & -0.04 & 0.02 & 0.03 & -0.11 \\
\hline 3. Positive Emotion Regulation & & & - & 0.23 & $-0.34^{*}$ & 0.22 & 0.10 & $-0.32^{*}$ \\
\hline 4. Negative Emotion Regulation & & & & - & 0.06 & 0.01 & 0.05 & 0.09 \\
\hline 5. Attention Problems & & & & & - & $0.62^{* *}$ & $0.50^{* *}$ & $0.74^{* *}$ \\
\hline 6. Emotional Reactivity & & & & & & - & $0.91^{* *}$ & $0.81^{* *}$ \\
\hline 7. Internalizing & & & & & & & - & $0.79^{*}$ \\
\hline 8. Externalizing & & & & & & & & - \\
\hline
\end{tabular}

Table 4. Intercorrelations among verbal ability, behavioral synchrony, emotion, and behavior problems for full-term children $(\mathrm{n}=28)$.

\begin{tabular}{|c|c|c|c|c|c|c|c|c|}
\hline & 1. & 2. & 3. & 4. & 5. & 6. & 7. & 8. \\
\hline 1. Verbal Ability & - & 0.33 & -0.10 & -0.12 & 0.10 & -0.13 & -0.05 & -0.05 \\
\hline 2. Behavioral Synchrony & & - & -0.22 & $0.63^{* *}$ & $0.46^{*}$ & 0.03 & 0.35 & 0.35 \\
\hline 3. Positive Emotion Regulation & & & - & 0.09 & -0.27 & $-0.04^{*}$ & -0.12 & -0.12 \\
\hline 4. Negative Emotion Regulation & & & & - & $-0.58^{* *}$ & -0.16 & -0.18 & $-0.59^{*}$ \\
\hline 5. Attention Problems & & & & & - & 0.12 & 0.28 & $0.75^{* *}$ \\
\hline 6. Emotional Reactivity & & & & & & - & $0.86^{* *}$ & $0.49^{* *}$ \\
\hline 7. Internalizing & & & & & & & - & $0.51^{* *}$ \\
\hline 8. Externalizing & & & & & & & & - \\
\hline
\end{tabular}

risk scores were correlated with verbal ability scores such that higher risk was correlated with lower verbal ability scores $(\mathrm{r}=-0.49 ; \mathrm{p}<0.01)$.

\subsection{Model Testing}

Hierarchical multiple regressions were used to test this study's main hypotheses and interaction effects in hierarchal regression were tested as recommended by Cohen and Cohen [21]. A series of hierarchal regressions were conducted to test the moderating effect of behavioral synchrony on the prediction of emotion regulation from verbal ability for both the LBW and full-term children separately as well as for the combined samples. Child's age was entered in the first step as covariates. The main effects of verbal ability and behavior synchrony 
were entered onto the second step, and the two-way interaction effects (verbal ability and behavior synchrony) were entered onto the third step. For the overall sample, the model predicting negative emotion regulation was significant, $\mathrm{R}=0.59, \mathrm{R} 2=0.29, \mathrm{~F}(5,61)=6.06, \mathrm{p}<0.001$, and the interaction term was significant, $\mathrm{B}=0.10, \mathrm{t}$ $=3.37, \mathrm{p}<0.001$. The interaction was examined at high, medium, and low levels of behavioral synchrony. Verbal ability significantly predicted negative emotion regulation scores at high levels of behavioral synchrony. At high levels of behavioral synchrony, as verbal ability increases, children's negative emotion regulation improves. When this regression model was conducted for the LBW sample, Hobel risk score was also entered in as a covariate. For the LBW group the model predicting emotion regulation was not significant $(p>0.05)$. For the full-term sample the overall model predicting negative emotion regulation was significant, $\mathrm{R}=0.74, \mathrm{R} 2=0.44$ $\mathrm{F}(5,26)=5.14, \mathrm{p}<0.001$, and the interaction term approached significance, $\mathrm{B}=0.15, \mathrm{t}=1.94, \mathrm{p}=0.07$.

\section{Discussion}

The purpose of the current research was to investigate the extent to which children's verbal ability and behavioral synchrony contribute to emotion regulation and behavior problems for preschool-age children. Research has indicated that for pre-term children, biological vulnerabilities impact the attainment of cognitive and emotion regulatory skills later in development [22] [23]. One way in which emotion regulatory skills are attained is through parental modeling and scaffolding of appropriate affect. Given the salience of caregivers for the development of children's emotion regulation, this study sought to examine how caregiver and child interactions impact the relations between verbal ability, emotion regulation and behavior problems for children at risk for emotion dysregulation and behavior problems.

As expected, the results revealed that the full-term children demonstrated higher verbal ability scores, however, in contrast to expectations, for LBW children, negative emotion regulation was not related to behaviors problems. Interestingly, the only group difference found in emotion expression was in the amount of joyful affect, with the full-term children demonstrating more joyful affect in response to caregiver interactions in a frustrating situation than the LBW children. This is particularly interesting in light of research on mother-preterm dyads that has found that preterm infants demonstrate duller or more blunted affect during interactions.

Analyses revealed significant correlations between verbal ability and behavior problems as well as behavioral synchrony and negative affect and emotion regulation. Based on the current findings, for LBW children, verbal ability plays a significant role in behavior problems but to a lesser extent for full-term children. These finding are in accord with current literature which asserts that language ability is related to children's behavior problems. [9] Of interest is that birth status and Hobel risk scores continues to impact verbal ability scores into the preschool years which supports research that asserts children with deficits in language development also demonstrate deficits in social understanding, [24] and social problem-solving [25] which continue later into development.

There are several limitations to the current study which impact findings. Much research has been conducted examining the cognitive and developmental difficulties in LBW families and this research replicates this, however, the findings may be pertinent to a limited demographic portion of the LBW population, namely European-American families. There may be parenting factors that differ amongst ethnic groups and these factors may impact the key variables of this research. The issue of length of the frustration task is also a factor in that it is possible that a longer period of frustration may increase generalizabilty of behaviors between laboratory and real-world settings and may account for the lack of detection of some difference amongst groups.

\section{Conclusions}

The findings suggest that children with better language skills may utilize language to support synchronous relationships that facilitate more positive emotion. The findings support research studies that assert that children with deficits in language development may experience deficits in other domains of functioning [26]. LBW children have been reported to have deficits in language development and emotion regulation due to the temperamental vulnerability for emotional lability and neonatal insults that impact cognitive functioning [27] and there is partial support for the relationship between verbal ability and emotion regulation in the current study.

This study supports the construct validity of emotion regulation by offering an additional unit of measurement besides the self-report measure to protect against issues of biases and measurement equivalence inherent in selfreport indices. Children's emotion regulation is often examined through post-hoc, parent administered, self- 
report measures. Within this method of measurement, biases emerge or issues of recollection impact parents' reports of child emotion regulation. Future research should examine whether parents' perceptions of emotion regulation differ from those of trained observers, and examine the convergent validity of this method of examining negative emotion regulation and reactivity, and other methods of measuring emotion regulation (e.g., paper and pencil forms) with respect to developing psychometrically sound methods of measurement.

Although support for the role of behavior synchrony was found in this study, future research will examine the manner in which synchrony develops particularly in high-risk infants as it has been demonstrated to be important to the dyadic relationship [28]-[30]. This research will be of importance as the current zeitgeist of empirically supported treatments for children with behavior disorders focuses on parent-child relationships, which serve to modify parental perceptions of behavior and impact dyadic relationship quality.

\section{References}

[1] Bradley, S. (1990) Affect Regulation and Psychopathology: Bridging the Mind-Body Gap. Canadian Journal of Psychiatry, 35, 540-547.

[2] Silk, J.S., Steinberg, L. and Morris, A. (2003) Adolescents' Emotion Regulation in Daily Fife: Links to Depressive Symptoms and Problem Behavior. Child Development, 74, 1869-1880. http://dx.doi.org/10.1046/j.1467-8624.2003.00643.x

[3] Calkins, S. and Johnson, M. (1998) Toddler Regulation of Distress to Frustrating Events: Temperamental and Maternal Correlates. Infant Behavior and Development, 21, 379-395. http://dx.doi.org/10.1016/S0163-6383(98)90015-7

[4] Feldman, R. (2006) From Biological Rhythms to Social Rhythms: Physiological Precursors to Mother-Infant Synchrony. Developmental Psychology, 42, 175-188. http://dx.doi.org/10.1037/0012-1649.42.1.175

[5] Eckerman, C., Hsu, H., Molitor, A., Leung, E. and Goldstein, R. (1999) Infant Arousal in an En-Face Exchange with a New Partner: Effects of Prematurity and Perinatal Biological Risk. Developmental Psychology, 35, 282-293. http://dx.doi.org/10.1037/0012-1649.35.1.282

[6] Sykes, D.H., Hoy, E.A., Bill, J.M., McClure, B.G., Halliday, H.L. and Reid, M.M. (1997) Behavioral Adjustment in School of Very Low Birthweight Children. Journal of Child Psychology and Psychiatry, 38, 315-325. http://dx.doi.org/10.1111/j.1469-7610.1997.tb01516.x

[7] Calkins, S. and Hill, A. (2007) Caregiver Influences on Emerging Emotion Regulation: Biological and Environmental Transactions in Early Development. In: Gross, J.J., Ed., Handbook of Emotion Regulation, Guilford Press, New York, 229-248.

[8] Adger-Antonikowski, A. Halpern, L.F., Gajee, N., Faust, A., Pugliano, D. and Lowe, K. (2005) Does Social Competence Moderate the Relation between Verbal Ability and Behavior Problems among High-Risk Pre-Schoolers. Proceedings The Society for Research in Child Development, Atlanta.

[9] Bowman, M., Barnett, D., Johnson, A. and Reeve, K. (2006) Language, School Functioning, and Behavior among African American Urban Kindergartners. Merrill Palmer Quarterly, 52, 216-238. http://dx.doi.org/10.1353/mpq.2006.0012

[10] Hart, K., Fujiki, M., Brinton, B. and Hart, C. (2004) The Relationship between Social Behavior and Severity of Language Impairment. Journal of Speech, Language, and Hearing, 47, 647-662. http://dx.doi.org/10.1044/1092-4388(2004/050)

[11] Mendez, J., Fantuzzo, J. and Cicchetti, D. (2002) Profiles of Social Competence among Low-Income African American Preschool Children. Child Development, 73, 1085-1100. http://dx.doi.org/10.1111/1467-8624.00459

[12] Nelson, J., Benner, G. and Cheney, D. (2005) An Investigation of the Language Skills of Students with Emotional Disturbance Served in Public School Settings. Journal of Special Education, 39, 97-105. http://dx.doi.org/10.1177/00224669050390020501

[13] Wulbert, M., Inglis, S., Kriegsmann, E. and Mills, B. (1975) Language Delay and Associated Mother-Child Interactions. Developmental Psychology, 11, 61-70. http://dx.doi.org/10.1037/h0076125

[14] Beckwith, L. and Cohen, S. (1989) Maternal Responsiveness with Preterm Infants and Later Competency. New Directions for Child Development, 43, 75-87. http://dx.doi.org/10.1002/cd.23219894308

[15] Lester, B., Hoffman, J. and Brazelton, T.B. (1985) The Rhythmic Structure of Mother-Infant Interaction in Term and Pre-Term Infants. Child Development, 56, 15-27. http://dx.doi.org/10.2307/1130169

[16] Halpern, L.F. and McLean, W.E. (1997) Hey Mom, Look at Me. Infant Behavior and Development, 20, 515-529. http://dx.doi.org/10.1016/S0163-6383(97)90040-0

[17] Landry, S.H., Smith, K.E., Swank, P.R. and Miller-Loncar, C.L. (2000) Ealry Maternal and Child Influences on Child- 
ren's Later Maladaptive Cogntive and Social Functioning. Child Development, 71, 358-375. http://dx.doi.org/10.1111/1467-8624.00150

[18] Taylor, H.B., Anthony, J.L., Aghara, R., Smith, K. and Landry, S.H. (2008) The Interaction of Early Maternal Responsiveness and Children's Cognitive Abilities on Later Decoding and Reading Comprehension Skills. Early Education and Development, 19, 188-207. http://dx.doi.org/10.1080/10409280701839304

[19] Thorndike, R., Hagen, E. and Sattler, J. (1996) Stanford-Binet Intelligence Scale. 4th Edition, Riverside Publishing Co., Rolling Meadows.

[20] Achenbach, T.N. (2000) Manual for the Child Behavior Checklist. University of Vermont Department of Psychiatry, Burlington.

[21] Cohen, J. and Cohen, P. (1983) Applied Multiple Regression/Correlation Analysis for the Behavioral Sciences. 2nd Edition, Erlbaum, Hillsdale.

[22] Alyward, G. (2003) Neonatology, Prematurity, NICU and Developmental Issues. In: Roberts, M., Ed., Handbook of Pediatric Psychology, Guilford Press, New York, 253-269.

[23] Nadeau, L., Boivin, M., Tessier, R., Lefebvre, F. and Robaey, P. (2001) Mediators of Behavioral Problems in 7Year-Old Children Born after 24 to 28 Weeks of Gestation. Journal of Developmental and Behavioral Pediatrics, 22, 1-10. http://dx.doi.org/10.1097/00004703-200102000-00001

[24] Fujiki, M., Spackman, M., Brinton, B. and Illig, T. (2008) Ability of Children with Language Impairment to Understand Emotion Conveyed by Prosody in a Narrative Passage. International Journal of Language and Communication Disorders, 43, 330-345. http://dx.doi.org/10.1080/13682820701507377

[25] Marton, K., Abramoff, B. and Rosenzweig, S. (2005) Social Cognition and Language in Children with Specific Language Impairment (SLI). Journal of Communication Disorders, 38, 143-162. http://dx.doi.org/10.1016/j.jcomdis.2004.06.003

[26] Kubicek, L., Emde, R. and Schmitz, S. (2001) Temperament, Mental Development, and Language in the Transition from Infancy to Early Childhood. In: Hewitt, J. and Emde, R., Eds, Infancy to Early Childhood: Genetic and Environmental Influences on Developmental Change, Oxford University Press, New York, 307-328.

[27] Le Norman, M. and Cohen, H. (1999) The Delayed Emergence of Lexical; Morphology in Preterm Children: The Case of Verbs. Journal of Neurolinguistics, 12, 235-246. http://dx.doi.org/10.1016/S0911-6044(99)00016-0

[28] Feldman, R. (2007) Mother-Infant Synchrony and the Development of Moral Orientation in Childhood and Adolescence: Direct and Indirect Mechanisms of Developmental Continuity. American Journal of Orthopsychiatry, 77, 582597. http://dx.doi.org/10.1037/0002-9432.77.4.582

[29] Feldman, R. and Klein, P.S. (2003) Toddlers' Self-Regulated Compliance to Mothers, Caregivers, and Fathers: Implications for Theories of Socialization. Developmental Psychology, 39, 680-692. http://dx.doi.org/10.1037/0012-1649.39.4.680

[30] Reck, C., Hunt, A., Fuchs, T., Weiss, R., Noon, A., Moehler, E., Downing, G., Tronick, E. and Mundt, C. (2004) Interactive Regulation of Affect in Postpartum Depressed Mothers and Their Infants: An Overview. Psychopathology, 37, 272-280. 